\title{
Impact of Mechanical Strain on GIFBE in PD SOI p-MOSFETs as Indicated From NBTI Degradation
}

\author{
Wen-hung Lo, Ting-Chang Chang, Chih-Hao Dai, Wan-Lin Chung, Ching-En Chen, \\ Szu-Han Ho, Osbert Cheng, and Cheng Tung Huang
}

\begin{abstract}
This letter investigates the impact of mechanical strain on gate-induced floating-body effect in partially depleted silicon-on-insulator p-channel metal-oxide-semiconductor fieldeffect transistors. The strained FB device has less NBTI degradation than unstrained devices. This behavior can be attributed to the fact that more electron accumulation induced by strain effect reduces the electric oxide field significantly during NBTI stress. Analysis of the body current $\left(I_{B}\right)$ under source/drain grounded and floating operation indicates an increase in the anode electron injection and electron tunneling from conduction band which occur at the partial $n^{+}$poly-Si gate and Si substrate, respectively. This phenomenon can be attributed to the bandgap narrowing which has been induced by the strain effect.
\end{abstract}

Index Terms-Gate-induced floating-body effect (GIFBE), negative bias temperature instability (NBTI), silicon-on-insulator (SOI) MOSFETs, strained silicon.

\section{INTRODUCTION}

$\mathbf{S}$ ILICON-on-insulator (SOI) CMOS devices are attractive for switching application because of their high speed, lower power dissipation, and ability to restrain latch-up. However, as devices are scaled down below $90 \mathrm{~nm}$, it becomes critical to realize the high drive current due to the degradation of carrier mobility caused by the required increase in channel doping [1]. Because of that, the use of a strained silicon technique offers an alternative method to enhance the SOI performance. In recent years, negative bias temperature instability (NBTI) has become a critical challenge due to the continuous shrinking of gate oxide thicknesses. Several studies have indicated that processinduced strain results in more serious NBTI degradation [2]-[4]. This is because forming gas may increase the number of $\mathrm{Si}-\mathrm{H}$ bonds and induce nitrogen incorporation, both of which aggravate NBTI [2], [5], [6]. In contrast, significant gate

Manuscript received November 7, 2011; accepted November 21, 2011. Date of publication January 23, 2012; date of current version February 23, 2012. The review of this letter was arranged by Editor M. Östling.

W.-h. Lo and W.-L. Chung are with the Department of Physics and the Institute of Electro-Optical Engineering, National Sun Yat-Sen University, Kaohsiung 80424, Taiwan.

T.-C. Chang is with the Department of Physics, the Department of Photonics, and the Center for Nanoscience and Nanotechnology, National Sun Yat-Sen University, Kaohsiung 80424, Taiwan (e-mail: tcchang@ mail.phys.nsysu.edu.tw).

C.-H. Dai is with the Department of Photonics, National Sun Yat-Sen University, Kaohsiung 80424, Taiwan.

C.-E. Chen and S.-H. Ho are with the Department of Electronics Engineering, National Chiao Tung University, Hsinchu 300, Taiwan.

O. Cheng and C. T. Huang are with the Device Department, United Microelectronics Corporation, Hsinchu 300, Taiwan.

Color versions of one or more of the figures in this letter are available online at http://ieeexplore.ieee.org.

Digital Object Identifier 10.1109/LED.2011.2177956 leakage current has been reported to induce a new floatingbody effect in SOI devices, called gate-induced floating-body effect (GIFBE) [7], [8], which could reduce NBTI degradation because of the lower oxide electric field during stress [9]-[11]. However, the influence of the strain effect on GIFBE has not yet been studied. Therefore, this letter investigates the impact of strain on GIFBE by analysis of NBTI behavior. Mechanical strain was used in this letter to avoid the forming gas process issues and therefore study the pure strain effect on GIFBE [12]-[14].

\section{EXPERIMENT}

Using 65-nm SOI CMOS technology, partially depleted SOI p-channel metal-oxide-semiconductor field-effect transistors (PD SOI p-MOSFETs) with $\mathrm{p}^{+}$poly-Si gate are produced in a T-gate structure. The SOI film and buried oxide thicknesses are 75 and $145 \mathrm{~nm}$, respectively. The gate oxide has a thickness of $12 \AA$, and the channel doping concentration is about $3 \times$ $10^{18} \mathrm{~cm}^{-3}$. The channel is along the $\langle 110\rangle$ direction on the (100) substrate. In this letter, devices have channel lengths $(L)$ of $0.2 \mu \mathrm{m}$ and widths $(W)$ of $1.0 \mu \mathrm{m}$. A stress gate voltage of $-1.8 \mathrm{~V}+V_{T}$ was applied to the gate electrode at $125^{\circ} \mathrm{C}$, while the source and drain were grounded. The threshold voltage $\left(V_{T}\right)$ was defined as the maximum of transconductor $\left(G_{m_{\max }}\right)$ in the linear region. All electrical characteristics were measured using an Agilent B1500 semiconductor parameter analyzer.

\section{Discussion AND Result}

The $I_{D}-V_{G}$ and $G_{m}-V_{G}$ characteristics are shown in the inset of Fig. 1(a). After uniaxial compressive strain, the device shows better performance. This phenomenon indicates that our proposed strain is valid on the device due to a smaller effective mass and longer scattering lifetime [15]. Based on this mechanical strain, Fig. 1(a) shows the NBTI-induced $V_{\text {th }}$ shift for the FB device before and after compressive strain. The $V_{\text {th }}$ shift for the FB device is less than that for the GB device. This phenomenon can be attributed to the electron accumulation in the body, reducing the oxide electric field during NBTI stress [9]-[11]. However, it is worth noting that, when applying strain to the FB devices, the NBTI degradation becomes less serious than in FB and GB conditions without strain. This is because mechanical strain induces more electron accumulation in the $\mathrm{FB}$, resulting in the significant lowering of the oxide electric field during NBTI stress. To further examine the physical origin of the degraded reliability resulting from the strain, the thermal activation energy $\left(E_{a}\right)$ of NBTI was evaluated, shown in Fig. 1(b). Several studies indicated that changes in the NBTI 

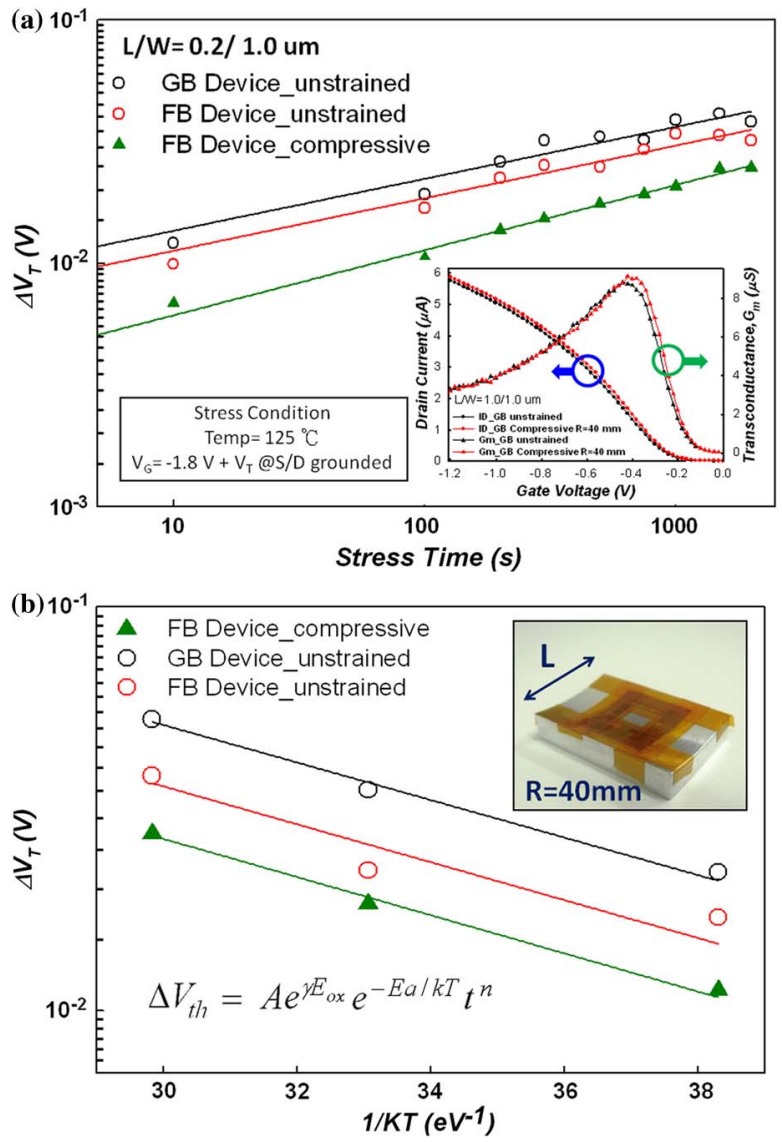

Fig. 1. (a) Time evolution of threshold voltage shift in SOI PD p-MOSFETs with unstrained and strained FB devices, in addition to unstrained GB device. The inset shows the $I-V$ curve of device before and after compressive strain. (b) Temperature dependence of threshold voltage shift of devices that are compressively strained and unstrained, with activation energy extracted from the power law with time under NBTI. The inset shows an illustration of bending sample with mechanical compressive strain.

mechanism can be observed by the changes in $E_{a}$ of NBTI [5], [6].

However, Fig. 1(b) shows a nearly unchanged $E_{a}$ of NBTI with strain $(\sim 0.11 \mathrm{eV})$. This suggests that the strain does not change the NBTI mechanism itself but only changes the amount of interface traps. These results evidence that the different levels of NBTI degradation in Fig. 1(a) can be purely attributed to the difference in oxide electric field induced by the amount of electron accumulation in the FB.

As described previously, the FB device has a decrease of electric field which is associated with electron accumulation in the body during NBTI. Furthermore, the amount of electron accumulation in the FB can be determined from measurement of the body current $\left(I_{B}\right)$ under GB condition. Therefore, to realize the influence of mechanical strain on NBTI under FB condition, the body current both with and without mechanical strain was measured and shown in Fig. 2. It can be seen that there is indeed an increase in $I_{B}$ under mechanical strain, further confirming that the strain induces more electron accumulation in the FB, resulting in less NBTI degradation for the FB device. In addition, previous studies have indicated that the $I_{G}$ can be effectively suppressed by applying compressive strain [16]. This slight reduction of gate current is due to strain-induced change in the valence band offset between $\mathrm{Si}$ and the $\mathrm{SiO}_{2}$ gate dielectric. Accordingly, the degree of NBTI degradation

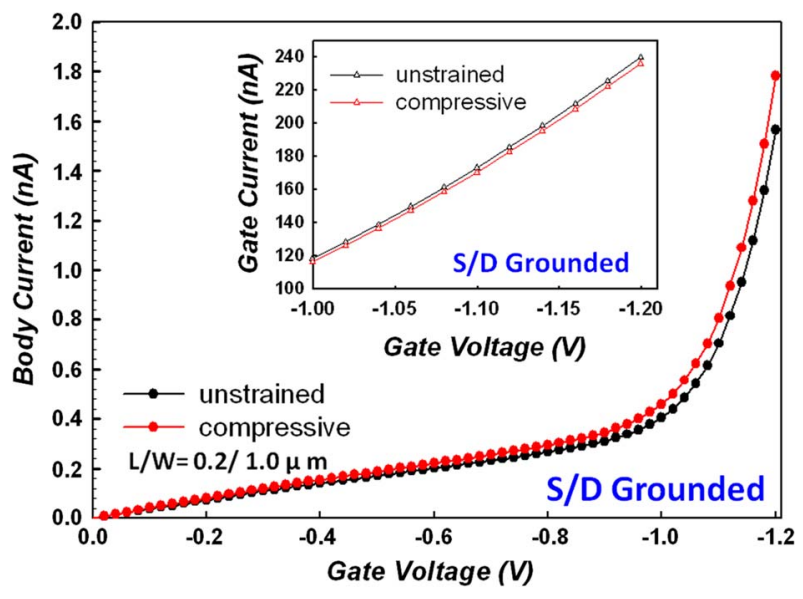

Fig. 2. Body current with S/D grounded for GB devices that are compressively strained and unstrained. The inset shows the gate current composed by source and drain current for GB devices that are compressively strained and unstrained.

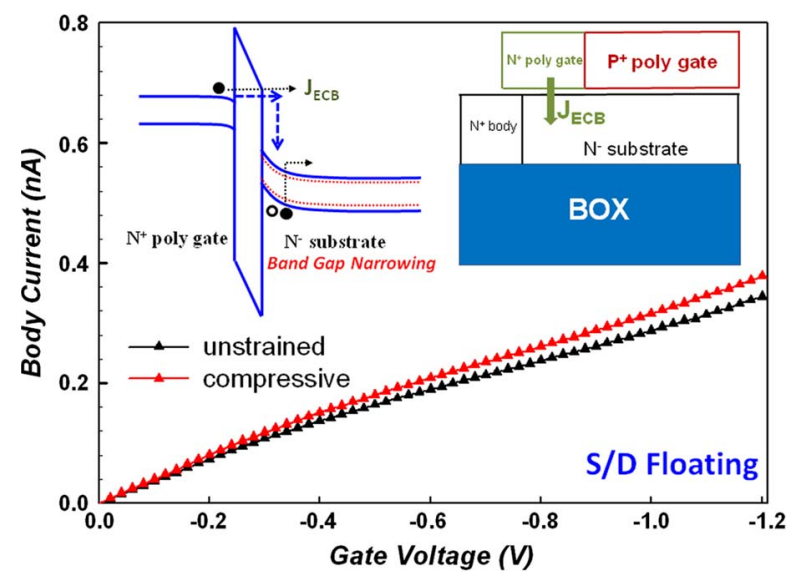

Fig. 3. $I_{B}-V_{G}$ curves with floating $\mathrm{S} / \mathrm{D}$ for strained and unstrained GB devices. The inset shows the schematic diagram of ECB from the partial $n^{+}$ poly-Si gate for an ultrathin gate oxide in PD SOI p-MOSFETs and a cross section of the device.

is closely related to the $I_{G}$ magnitude, which is consistent with the probability of reaction of the holes and $\mathrm{Si}-\mathrm{H}$ bonds [17]. Experimental results indicate that the GB device has an improvement of about 5.3\% in NBTI reliability after strain (not shown here). However, there is a significant improvement in NBTI reliability of about $22.8 \%$ for the FB device. Therefore, this additional improvement can be attributed to the lowering of the oxide electric field by more electron accumulation.

The two stages of increase in $I_{B}$, as shown in Fig. 2, can be attributed to the electron tunneling from conduction band (ECB) and anode electron injection (AEI) mechanisms, respectively [9]. The ECB tunneling is induced by the partial $\mathrm{n}^{+}$poly-Si of the body-tied device, as shown in the inset of Fig. 3. The AEI is associated with the inversion layer supplied by the source/drain (S/D). The holes tunnel from the inversion layer and generate hot electrons by impact ionization in the poly-Si depletion region, resulting in the second increase of $I_{B}$. Fig. 2 shows that there is an increase in both stages of $I_{B}$ under the strain condition. To clearly examine the influence of strain effect on the first stage of $I_{B}$, ECB tunneling before and after strain was measured under floating S/D operation and shown in Fig. 3. There is an increase in $I_{B}$, with gate bias increasing 


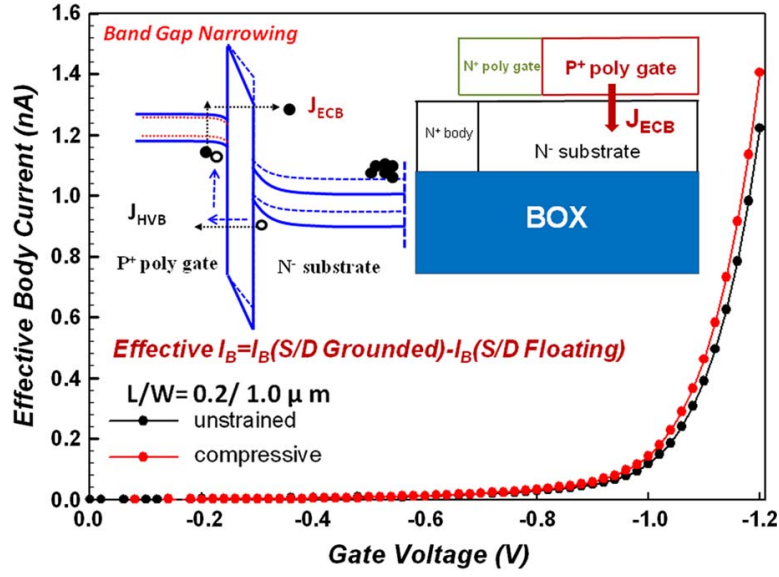

Fig. 4. Comparison of effective body current versus gate voltage for compressive strained and unstrained devices. The inset shows the cross section and the schematic diagram of AEI mechanism under strain.

beyond the $V_{G}$ of $-0.4 \mathrm{~V}$. In addition, the difference of $I_{B}$ between unstrained and strained devices becomes significant with increasing $V_{G}$. Electrons tunneling from the poly-Si gate still can generate hot holes by impact ionization in the depletion region of an n-type substrate. This suggests that the first stage of $I_{B}$ cannot be purely attributed to ECB tunneling but still reflects the contribution of hot holes by impact ionization in the n-type substrate, as shown in the inset of Fig. 3. Therefore, we suggest that the increase of $I_{B}$ in Fig. 3 is mainly due to the bandgap narrowing of the n-type substrate induced by the mechanical strain effect. This phenomenon reduces the threshold energy to generate electron-hole pairs by impact ionization, resulting in the first stage increase of $I_{B}$ after strain. Therefore, with an increase in gate bias, the difference of $I_{B}$ before and after strain gradually becomes clear, as shown in Fig. 3. However, in Fig. 2, the second stage of $I_{B}$ also increases under mechanical strain, a phenomenon which may be related to the first stage increase of $I_{B}$. To clearly distinguish the contribution of the strain effect on the second stage of $I_{B}$, the pure AEI-induced electron current (effective $I_{B}$ ) was also obtained, as shown in Fig. 4, by subtracting the ECB tunneling component from the second increase of $I_{B}$. Based on the AEI model, the $I_{B}$ generally decreases as the $I_{G}$ decreases. However, the strain effect increases the effective $I_{B}$, particularly over $V_{G}=-1 \mathrm{~V}$. This unusual phenomenon is caused by the bandgap narrowing induced by the mechanical strain effect. Because the impact ionization rate is exponential to the energy bandgap, the narrowing bandgap causes more additional electron generation in the poly-Si depletion region by impact ionization even if $I_{G}$ decreases.

\section{CONCLUSION}

This letter has investigated the influence of mechanical strain on GIFBE from NBTI degradation in PD SOI p-MOSFETs. It is found that the strained device under FB operation exhibits much less NBTI degradation. This is because the higher electron accumulation induced by strain effect has reduced the electric oxide field during NBTI stress. These electrons are generated by the ECB and AEI mechanisms. Based on our systematical analysis, strain effect induces an increase in two stages of $I_{B}$. This can be attributed to bandgap narrowing in $\mathrm{n}^{-}$substrate and $\mathrm{p}^{+}$poly-Si gate, respectively.

\section{ACKNOWLEDGMENT}

Part of this work was performed at United Microelectronics Corporation.

\section{REFERENCES}

[1] T. Ghani, K. Mistry, P. Packan, S. Thompson, M. Stealer, and S. Tyagi, "Scaling challenges and device design requirements for high performance sub-50 nm gate length planar CMOS transistors," in VLSI Symp. Tech Dig, 2000, pp. 174-175.

[2] H. S. Rhee, H. Lee, T. Ueno, D. S. Shin, S. H. Lee, Y. Kim, A. Samoilov, P. O. Hansson, M. Kim, H. S. Kim, and N. I. Lee, "Negative bias temperature instability of carrier-transport enhanced pMOSFET with performance boosters," in IEDM Tech. Dig., 2005, pp. 692-695.

[3] G. Thareja, F. Z. J. Lee, A. V. Y. Thean, V. Vartanian, and B. Y. Nguyen, "NBTI reliability of strained SOI MOSFETs," in Proc. ISTFA, 2006, pp. 423-425.

[4] Y. W. Jeon, D. H. Ka, C. G. Yu, W. J. Cho, M. S. Islam, and J. T. Park, "NBTI and hot carrier effect of SOI p-MOSFETs fabricated in strained Si SOI wafer," Microelectron. Reliab., vol. 49, no. 9-11, pp. 994-997, Sep.-Nov. 2009.

[5] Y. Mitani, M. Nagamine, H. Satake, and A. Toriumi, "NBTI mechanism in ultra-thin gate dielectric - nitrogen-originated mechanism in $\mathrm{SiON}$," in IEDM Tech. Dig., 2002, pp. 509-512.

[6] N. Kimizuka, K. Yamaguchi, K. Imai, C. T. Liu, R. C. Keller, and T. Horiuchi, "NBTI enhancement by nitrogen incorporation into ultrathin gate oxide for 0.10- $\mu \mathrm{m}$ gate CMOS generation," in VLSI Symp. Tech. Dig., 2000, pp. 92-93.

[7] J. Pretet, T. Matsumoto, T. Poiroux, S. Cristoloveanu, R. Gwoziecki, C. Raynaud, A. Roveda, and H. Brut, "New mechanism of body charging in partially depleted SOI-MOSFETs with ultra-thin gate oxides," in Proc. ESSDERC, 2002, pp. 515-518.

[8] M. Casse, J. Pretet, S. Cristoloveanu, T. Poiroux, C. Fenouillet-Beranger, F. Fmleux, C. Raynaud, and G. Reimbold, "Gate-induced floating-body effect in fully-depleted SOI MOSFETs with tunneling oxide and backgate biasing," Solid State Electron., vol. 48, no. 7, pp. 1243-1247, Jul. 2004.

[9] C. H. Dai, T.-C. Chang, A.-K. Chu, Y.-J. Kuo, F.-Y. Jian, W.-H. Lo, S.-H. Ho, C.-E. Chen, W.-L. Chung, J.-M. Shih, G. Xia, O. Cheng, and C.-T. Huang, "On the origin of gate-induced floating-body effect in PD SOI p-MOSFETs," IEEE Electron Device Lett., vol. 32, no. 7, pp. 847849, Jul. 2011.

[10] R. Mishra, D. E. Ioannou, S. Mitra, and R. Gauthier, "Effect of floating-body and stress bias on NBTI and HCI on 65-nm SOI pMOSFETs," IEEE Electron Device Lett., vol. 29, no. 3, pp. 262-264, Mar. 2008

[11] J. Zhang, A. Marathe, K. Taylor, E. Zhao, and B. En, "New findings of NBTI in partially depleted SOI transistors with ultra-thin gate dielectrics," in Proc. Int. Reliab. Phys. Symp., 2004, pp. 687-688.

[12] Y. J. Kuo, T. C. Chang, P. H. Yeh, S. C. Chen, C. H. Dai, C. H. Chao, T. F. Young, O. Cheng, and C. T. Huang, "Substrate current enhancement in $65 \mathrm{~nm}$ metal-oxide-silicon field-effect transistor under external mechanical stress," Thin Solid Films, vol. 517, no. 5, pp. 1715-1718, Jan. 2009.

[13] C. H. Dai, T. C. Chang, A. K. Chu, Y. J. Kuo, S. C. Chen, C. T. Tsai, W. H. Lo, S. H. Ho, G. Xia, O. Cheng, and C. T. Huang, "Enhanced gate-induced floating-body effect in PD SOI MOSFET under external mechanical strain," Surf. Coatings Technol., vol. 205, no. 5, pp. 14701474, Nov. 2010

[14] C. S. Lin, Y.-C. Chen, T.-C. Chang, F.-Y. Jian, W.-C. Hsu, Y.-J. Kuo, C.-H. Dai, T.-C. Chen, W.-H. Lo, T.-Y. Hsieh, and J.-M. Shih, "NBTI degradation in LTPS TFTs under mechanical tensile strain," IEEE Electron Device Lett., vol. 32, no. 7, pp. 907-909, Jul. 2011.

[15] C. W. Liu, S. Maikap, and C. Y. Yu, "Mobility-enhancement technologies," IEEE Circuits Devices Mag., vol. 21, no. 3, pp. 21-36, May/Jun. 2005

[16] X. Yang, J. Lim, G. Sun, K. Wu, T. Nishida, and S. E. Thompson, "Strain-induced changes in the gate tunneling currents in p-channel metal-oxide-semiconductor field-effect transistors," Appl. Phys. Lett., vol. 88, no. 5, p. 052108 , Jan. 2006.

[17] T. Irisawa, T. Numata, E. Toyoda, N. Hirashita, T. Tezuka, N. Sugiyama, and S. I. Takagi, "Physical understanding of strain-induced modulation of gate oxide reliability in MOSFETs," IEEE Trans. Electron Devices, vol. 55, no. 11, pp. 3159-3166, Nov. 2008. 